\title{
Depression among type 2 diabetic patients
}

\author{
Taghreed Mohamed El-Shafie* ${ }^{*}$ Entesar Omar A. El-Saghier** and Iman \\ Kamal Ramadan*** \\ Psychiatry Department *, Endocrinology Department ** and Community \\ Department****, Faculty of Medicine for Girls, Al-Azhar University
}

\begin{abstract}
Background \& Objectives: Diabetes and depression are highly prevalent conditions and have significant impact on health outcomes. The combination of depression with type 2 diabetes is a public health problem. Therefore, we aimed to assess some socio-demographic characteristics of type 2 diabetes and to investigate the relationship between type 2 diabetes and depression among patients aged from 40 to 60 years old.
\end{abstract}

Methods: 125 patients diagnosed with type 2 diabetes attending diabetes clinics in the AlZahraa hospital were invited to participate in this cross-sectional study. Patients were interviewed using structured questionnaires to gather data on socio-demographics, clinical, self care compliance, medication usage, and diabetes complications. The MADRS was administered as a screening tool for depression level evaluation. Binary logistic regression model was used to examine association between predictor variables and risk of depression among diagnosed type 2 diabetes at $95 \%$ C.I. and $\mathrm{P}<0.05$.

Results: One hundred and twenty five participants completed the interview. More than half of participants were females $(58.4 \%)$ and the mean age was $48(\mathrm{sd}=5.9), 47.2 \%$ hypertensive, and $59.2 \%$ on insulin. More than two third $(74.4 \%)$ of patients were depressed; (24.8\% mild, $37.6 \%$ moderate and $12 \%$ severely depressed). Almost four out of five patients $(88.8 \%)$ had diabetes complications, Depression was strongly associated with neuropathy, age, retinopathy, sex and cardiac complications. However, the likelihood of depression was not associated with nephropathy, hypertension and sexual dysfunction.

Conclusion: The current study demonstrates a strong correlation between depression and diabetes particularly complications. In particular, patients who are depressed tended to have poorer self-care, more severe physical symptoms and were less likely to adhere to prescribed care regimens. These findings raise the possibility that improving the mental health as part of a comprehensive management plan for diabetes may improve the overall long term outcomes of these patients.

Key Words: depression • diabetes mellitus. MADRS

\section{Introduction}

Associations between depression and diabetes have been described by physicians for several hundred years. Thomas Willis, an early English physician and anatomist, wrote during the mid-1600s that, diabetes is caused by "sadness or long sorrow and other depressions ".In recent years; researchers have posited that there is a bidirectional link between depression and diabetes (Katon, 2010).
$\mathrm{DM}$ is a major emerging clinical public and health problem in Egypt (Herman et al., 1997). The combined prevalence of diagnosed and undiagnosed diabetes in the Egyptian population 20 years of age was 9.3 (Herman et al., 1995).

By the 2025, Egypt is expected to be among the top ten country that have the highest prevalence rates of diabetes in the world, notably Type 2 diabetes mellitus (T2DM) (King et al 1998). Also it is 
expected that, 13.3 of the population 20 years age will have DM, the elderly will represent $21 \%$ of the total population with diabetes and urban residents will represent 82\% (Herman et al., 1997). This increase in percentage of DM may be due to that; Egypt has experienced population growth and aging and has become more urban and affluent (Government of Egypt, Ministry of Health, Information Center, 1994) This affluence has lead to the adoption of unhealthy lifestyle resulting in a surge of chronic ill-health e.g DM. In rural areas, $52 \%$ of persons were sedentary, $16 \%$ obese and $4.9 \%$ had DM. However, in higher SES urban areas, $89 \%$ were sedentary, $49 \%$ obese and $20 \%$ had DM (Herman et al., 1995).

World Health Organization WHO ranked depression the fourth global burden of disease and found it to be the largest nonfatal burden of disease, with nearly $12 \%$ of total years lived with disability (Timonen and Liukkonen, 2008). It is predicted to become second only to ischemic heart disease as a cause of morbidity worldwide (Lester and Howe, 2008). In Egypt, the prevalence of depression among a selected sample of urban and rural population was found to be $11.4 \%$ and $19.7 \%$ respectively (Okasha, 2005).

Patients with type $2 \mathrm{DM}$ have a rate of major depression 1.6-2 times higher than those in the general population (Markowitz et al., 2011), affecting one of every 10 diabetic patients (Pouwer et al., 2010). Life time rates of major depression in type 2 diabetics are between $24 \%$ and 29\% (Geffken et al., 1998), and point prevalence is $10-15 \%$ (Fisher et al., 2008).

Depression is adversely associated with diabetes, from incidence to mortality (Lin et al., 2009). Compared with individuals with diabetes alone those with co-morbid depression have increased disease burden, greater symptoms severity, increased work disability, poorer adherence to diet, to exercise, to anti-diabetic, lipid lowering, and antihypertensive treatment (Gonzalez et al., 2007).
Developing countries like Egypt still have insufficient data on association of depression with type 2 diabetes. The current study therefore, assesses some socio-demographic characteristics of T2DM, and investigates the association of depression with diagnosed type 2 diabetes among diabetics aged between 40 to 60 years in Cairo, Egypt.

\section{Subjects and Methods}

The study was conducted at the specialist endocrine and psychiatry clinics of AlZahraa University Hospital which is a tertiary care hospital that caters mostly to middle and lower socio-economic strata

\section{Study design and Population}

A cross sectional study was conducted from July 2010 to December 2010. All consecutive patients having type 2 diabetes and its related complications between 40 and 60 years of age who had at least 2 clinic visits for management of diabetes mellitus were selected. Exclusion criteria were those having history of psychiatric illnesses other than depression, and who were currently on anti-depressant treatment and/or those who had history of use of psychotropic drugs, pregnancy, liver disease, advanced diabetic complications "blindness, renal failure, diabetic foot , or amputation".

The sample size was calculated to be 125 , keeping beta error at 0.2 significance level 0.05 and it was collected randomly.

\section{Measures}

Nursing assessment was performed for each participant that included recording of blood pressure, height and weight. After an informed consent, a structured clinical interview was performed by the researchers before each participant's consultation with the endocrinologist. Privacy was maintained throughout the interview. Details of each participant's demographics [age, sex, marital status, social class and smoking], family history of diabetes/depression, type of treatment, number of pills \& symptoms of diabetes and compliance to self-care activities were recorded. 
All consecutive type 2 diabetic patients were checked for presence of diabetesrelated complications; retinopathy "fundus examination", neuropathy "history and clinical examination", nephropathy "Micral test and confirmed by albumin creatinin ratio", cardiovascular diseases "history of angina, myocardial infarction or documented by previous treatment records, resting ECG, Echocardiography also done", sexual dysfunction "weakness and /or inability to maintain penil erection".

Blood was drown after overnight fasting for 8 hours for measurement of serum creatinine and $\mathrm{HbAlc}$ (quantitative colorimetric determination of glycolhemoglobin in whole blood).

The nine-item version of the SelfCompletion Patient Outcome instrument; measured the following diabetes symptoms: cold hands and feet, numb hands and feet, polyuria, excessive hunger, abnormal thirst, shakiness, blurred vision, feeling faint and feeling sleepy. Items were rated on a Likert scale from "never" to "every day." (Whitty et al., 1997).

Psychiatric assessment for all patients through psychiatric interview and Montgomery Asberg Depression Rating Scale (MADRS) (Montgomery and Asberg, 1979). Those who screened negative for depression where decided to be control group.

MADRS is a rating scale for assessment of depression and consists of 10 items, which are rated on a $0-6$ scale $(0=$ no abnormality \& $6=$ severe $)$. The items are; apparent sadness, reported sadness, inner tension, concentration difficulties, Lassitude, inability to feel, pessimistic thoughts and suicidal thoughts.

The Arabic version was done by the department of psychiatry of Al-Zahraa University Hospital.

All patients including cases \& control group subjected to the following assessment:

For obesity and overweight, Body Mass Index (BMI) at the Asian cut off was measured (underweight: $<18.50 \mathrm{~kg} / \mathrm{m} 2$, healthy: 18.50 to $22.90 \mathrm{~kg} / \mathrm{m} 2$, overweight: 23.00 to $24.90 \mathrm{~kg} / \mathrm{m} 2$, obese: $>25.00 \mathrm{~kg} / \mathrm{m} 2)$, by height scale \& calibrated bathroom scales.
Glycosylated Hemoglobin (HbA1c) was used as a measure of glycemic control $(<7 \%$ good compliance; $\geq 7 \%$ poor compliance) (ADA, 2009).

Compliance to self-care activities was measured using a self-report technique inquiring on treatment (timing/dosing), diet, blood glucose checking as advised, and care of feet. (Saman et al., 2001).

Systolic and diastolic blood pressure (SBP \& DBP) was measured twice at an interval of $3 \mathrm{~min}$ in the sitting position after a 15 min rest, and the mean was taken.

Study methods were approved from the Al-Zahraa University Ethics Research Committee.

\section{Statistical analysis}

Frequencies of all demographic and clinical variables were computed to identify sample characteristics. Body Mass Index (BMI) was computed using standard formula. Quantitative variables such as age, duration of illness, BMI, and $\mathrm{Hb} \mathrm{A} 1 \mathrm{c}$ were regrouped in equally distributed categories.

Differences in characteristics between participants were tested with the chisquare test for categorical variables. The significance level was set at $5 \%$.

Binary logistic regression model was used to examine association between predictor variables and risk of depression. The main model consists of following predictor variables: demographic variables age, sex, and diabetes complications "neuropathy, nephropathy, retinopathy, cardiac complications and sexual dysfunction" and hypertension. Statistical Package for Social Sciences Version 17.0 was used for these analyses.

\section{Results}

One hundred and twenty five completed questionnaires of type 2 diabetic patients were analyzed, their mean age $48 \pm 5.9$ years, females constituted $58.4 \%$ of the study sample, more than half the studied sample were married (66/125, 52.8\%), nearly half the sample were belonged to middle social class $(59 / 125,47.2 \%)$. 
Table 1 shows that severe depression was significantly higher in women than men ( $86.7 \%$ vs. $13.3 \%$; $p=0.001)$. However, there was no statistically significant difference regarding marital status, and social class.

Table 2 shows that the relation between duration of diabetes and depression, there was significant difference ( $p$ value 0.000). More than half type 2 diabetics (74/125, $59.2 \%$ ), were using insulin compared to $40.8 \%$ who used oral hypoglycemic drugs. All insulin users' type 2 diabetic patients were severely depressed. The difference was found to be statistically significant ( $p$ value 0.002).

$86.7 \%$ of severely depressed type 2 diabetics were consuming five pills or more compared to only $13.3 \%$ who consumed less than five pills and this difference is statistically significant $(\mathrm{p}=$ $0.02)$.

Depressed patients were more likely to have poor glycemic control than nondepressed patients. The mean $\mathrm{HbA1c}$ level was significantly higher in mild, moderate and severely depressed respectively than non-depressed patients ( $\mathrm{p}$ value 0.000 ).

Regarding compliance to self care, medication and preventive care, depressed type 2 diabetics were significantly less practicing self-care activities, $(P=0.45$, 0.07). Also, it should be noted that a higher level of physical inactivity was found among depressed type 2 diabetics, this relationship was significant $(p=$ 0.000).

Almost four out of five patients $(\mathrm{N}=$ $111 / 125,88.8 \%$ ) had a diabetes-related complications. More; mild, moderate and severely depressed patients reported a diabetes-related complication than nondepressed patients $(P=0.000)$. All these findings were illustrated in table 3 .

In relation to diabetes-related complications, many variables were found to be significantly related to MADRS scores. These variables were, neuropathy, retinopathy, nephropathy and cardiac complications $(\mathrm{p}=0.00)$.

Around half of type 2 diabetic patients had concomitant hypertension and $80 \%$ of severely depressed patients were hypertensive, therefore, it was statistically significant ( $p$ value 0.021).

Similarly, apart from sexual dysfunction, $80 \%$ of severely depressed type 2 diabetics were sexually dys-functioning and this was statistical significant difference $(\mathrm{p}=$ 0.00 ). These results showed in table 4

Table 5 summarizes the results of the multivariate logistic regression analyses. Out of all the variables included in the model, the only significant predictors of depression were found to be neuropathy, age , retinopathy , and sex 
(Table: 1) Socio-demographic characteristics of T2DM studied group

\begin{tabular}{|c|c|c|c|c|c|c|c|c|c|c|}
\hline Depression & \multicolumn{2}{|c|}{ Normal } & \multicolumn{2}{|c|}{ Mild } & \multicolumn{2}{|c|}{ Moderate } & \multicolumn{2}{|c|}{ Severe } & \multirow{2}{*}{$\begin{array}{c}\text { Chi- } \\
\text { Square }\end{array}$} & \multirow{2}{*}{$\begin{array}{c}\mathbf{P} \\
\text { value }\end{array}$} \\
\hline Variable & No & $\%$ & No & $\%$ & No & $\%$ & No & $\%$ & & \\
\hline $\begin{array}{l}\text { Age } \\
<50 \text { years } \\
\geq 50 \text { years }\end{array}$ & $\begin{array}{l}16 \\
16\end{array}$ & $\begin{array}{l}50 \\
50\end{array}$ & $\begin{array}{l}21 \\
10\end{array}$ & $\begin{array}{l}67.7 \\
32.3\end{array}$ & $\begin{array}{l}43 \\
4\end{array}$ & $\begin{array}{l}91.5 \\
8.5\end{array}$ & $\begin{array}{l}15 \\
0\end{array}$ & $\begin{array}{l}100 \\
0\end{array}$ & 23.94 & 0.00 \\
\hline $\begin{array}{l}\text { Sex } \\
\text { Male } \\
\text { Female }\end{array}$ & $\begin{array}{l}22 \\
10\end{array}$ & $\begin{array}{l}68.8 \\
31.3\end{array}$ & $\begin{array}{l}12 \\
19\end{array}$ & $\begin{array}{l}38.7 \\
61.3\end{array}$ & $\begin{array}{l}16 \\
31\end{array}$ & $\begin{array}{l}34 \\
66\end{array}$ & $\begin{array}{l}2 \\
13\end{array}$ & $\begin{array}{l}13.3 \\
86.7\end{array}$ & 15.85 & 0.001 \\
\hline $\begin{array}{l}\text { Marital status } \\
\text { Single } \\
\text { Married } \\
\text { Divorced } \\
\text { Widow }\end{array}$ & $\begin{array}{l}4 \\
20 \\
4 \\
4\end{array}$ & $\begin{array}{l}12.5 \\
62.5 \\
12.5 \\
12.5\end{array}$ & $\begin{array}{l}3 \\
18 \\
6 \\
4\end{array}$ & $\begin{array}{l}9.7 \\
58.1 \\
19.4 \\
12.9\end{array}$ & $\begin{array}{l}7 \\
22 \\
11 \\
7\end{array}$ & $\begin{array}{l}14.9 \\
46.8 \\
23.4 \\
14.9\end{array}$ & $\begin{array}{l}0 \\
6 \\
8 \\
1\end{array}$ & $\begin{array}{l}0 \\
40 \\
53.3 \\
6.7\end{array}$ & 12.14 & 0.21 \\
\hline $\begin{array}{l}\text { Social class } \\
\text { Low } \\
\text { Middle }\end{array}$ & $\begin{array}{l}16 \\
16\end{array}$ & $\begin{array}{l}50 \\
50\end{array}$ & $\begin{array}{l}17 \\
14\end{array}$ & $\begin{array}{l}54.8 \\
45.2\end{array}$ & $\begin{array}{l}24 \\
23\end{array}$ & $\begin{array}{l}51.1 \\
48.9\end{array}$ & $\begin{array}{l}9 \\
6\end{array}$ & $\begin{array}{l}60 \\
40\end{array}$ & 0.52 & 0.91 \\
\hline $\begin{array}{l}\text { Family history of } \\
\text { diabetes } \\
\text { - ve } \\
+ \text { ve }\end{array}$ & $\begin{array}{l}14 \\
18\end{array}$ & $\begin{array}{l}43.8 \\
56.3\end{array}$ & $\begin{array}{l}12 \\
19\end{array}$ & $\begin{array}{l}38.7 \\
61.3\end{array}$ & $\begin{array}{l}11 \\
36\end{array}$ & $\begin{array}{l}23.4 \\
76.5\end{array}$ & $\begin{array}{l}0 \\
15\end{array}$ & $\begin{array}{l}0 \\
100\end{array}$ & 11.48 & 0.009 \\
\hline $\begin{array}{l}\text { Family history of } \\
\text { depression } \\
\text { - ve } \\
+ \text { ve }\end{array}$ & $\begin{array}{l}32 \\
0\end{array}$ & $\begin{array}{l}100 \\
0\end{array}$ & $\begin{array}{l}31 \\
0\end{array}$ & $\begin{array}{l}100 \\
0\end{array}$ & $\begin{array}{l}43 \\
4\end{array}$ & $\begin{array}{l}91.5 \\
8.5\end{array}$ & $\begin{array}{l}9 \\
6\end{array}$ & $\begin{array}{l}60 \\
40\end{array}$ & 26.36 & 0.00 \\
\hline $\begin{array}{l}\text { Smoking } \\
\text { Non smokers } \\
\text { Smokers }\end{array}$ & $\begin{array}{l}26 \\
6\end{array}$ & $\begin{array}{l}81.3 \\
18.7\end{array}$ & $\begin{array}{l}23 \\
8\end{array}$ & $\begin{array}{l}74.2 \\
25.8\end{array}$ & $\begin{array}{l}31 \\
16\end{array}$ & $\begin{array}{l}66 \\
34\end{array}$ & $\begin{array}{l}6 \\
9\end{array}$ & $\begin{array}{l}40 \\
60\end{array}$ & 8.70 & 0.03 \\
\hline $\begin{array}{l}\text { BMI } \\
\text { Ideal } \\
\text { Overweight } \\
\text { Obese }\end{array}$ & $\begin{array}{l}1 \\
23 \\
8\end{array}$ & $\begin{array}{l}3.1 \\
71.9 \\
25\end{array}$ & $\begin{array}{l}0 \\
1 \\
30\end{array}$ & $\begin{array}{l}0 \\
3.2 \\
96.8\end{array}$ & $\begin{array}{l}1 \\
2 \\
44\end{array}$ & $\begin{array}{l}2.1 \\
4.3 \\
93.6\end{array}$ & $\begin{array}{l}1 \\
0 \\
14\end{array}$ & $\begin{array}{l}6.7 \\
0 \\
93.3\end{array}$ & 71.06 & 0.00 \\
\hline $\begin{array}{l}\text { Waist circumference } \\
<90 \mathrm{~cm} \\
\geq 90 \mathrm{~cm}\end{array}$ & $\begin{array}{l}30 \\
2\end{array}$ & $\begin{array}{l}93.8 \\
6.2\end{array}$ & $\begin{array}{l}15 \\
16\end{array}$ & $\begin{array}{l}48.4 \\
51.6\end{array}$ & $\begin{array}{l}18 \\
29\end{array}$ & $\begin{array}{l}38.3 \\
61.7\end{array}$ & $\begin{array}{l}0 \\
15\end{array}$ & $\begin{array}{l}0 \\
100\end{array}$ & 42.10 & 0.00 \\
\hline
\end{tabular}

Table 1 shows that the difference was statistically significant ( $p$ value 0.000)., 
Depression among....

(Table: 2) Clinical characteristics of T2DM studied group

\begin{tabular}{|c|c|c|c|c|c|c|c|c|c|c|}
\hline Depression & \multicolumn{2}{|c|}{ Normal } & \multicolumn{2}{|c|}{ Mild } & \multicolumn{2}{|c|}{ Moderate } & \multicolumn{2}{|c|}{ Severe } & \multirow{2}{*}{$\begin{array}{l}\text { Chi- } \\
\text { Square }\end{array}$} & \multirow{2}{*}{$\begin{array}{c}\mathbf{P} \\
\text { value }\end{array}$} \\
\hline Variable & No & $\%$ & No & $\%$ & No & $\%$ & No & $\%$ & & \\
\hline $\begin{array}{l}\text { Diabetes duration } \\
<8 \text { years } \\
\geq 8 \text { years }\end{array}$ & $\begin{array}{l}25 \\
7 \\
\end{array}$ & $\begin{array}{l}78.1 \\
21.9 \\
\end{array}$ & $\begin{array}{l}20 \\
11\end{array}$ & $\begin{array}{l}64.5 \\
35.5 \\
\end{array}$ & $\begin{array}{l}26 \\
21\end{array}$ & $\begin{array}{l}55.3 \\
44.7 \\
\end{array}$ & $\begin{array}{l}0 \\
15\end{array}$ & $\begin{array}{l}0 \\
100 \\
\end{array}$ & 26.45 & 0.00 \\
\hline $\begin{array}{l}\text { Number of } \\
\text { symptoms } \\
<3 \text { symptoms } \\
\geq 3 \text { symptoms }\end{array}$ & $\begin{array}{l}32 \\
0\end{array}$ & $\begin{array}{l}100 \\
0\end{array}$ & $\begin{array}{l}20 \\
11\end{array}$ & $\begin{array}{l}64.5 \\
35.5\end{array}$ & $\begin{array}{l}21 \\
26\end{array}$ & $\begin{array}{l}44.7 \\
55.3\end{array}$ & $\begin{array}{l}0 \\
15\end{array}$ & $\begin{array}{l}0 \\
100\end{array}$ & 47.97 & 0.00 \\
\hline $\begin{array}{l}\text { Type of treatment } \\
\text { Insulin } \\
\text { Oral }\end{array}$ & $\begin{array}{l}14 \\
18\end{array}$ & $\begin{array}{l}43.8 \\
56.3\end{array}$ & $\begin{array}{l}16 \\
15\end{array}$ & $\begin{array}{l}51.6 \\
48.4\end{array}$ & $\begin{array}{l}29 \\
18\end{array}$ & $\begin{array}{l}61.7 \\
38.3\end{array}$ & $\begin{array}{l}15 \\
0\end{array}$ & $\begin{array}{l}100 \\
0\end{array}$ & 14.36 & 0.002 \\
\hline $\begin{array}{l}\text { Number of pills } \\
<5 \text { pills } \\
\geq 5 \text { pills }\end{array}$ & $\begin{array}{l}20 \\
12 \\
\end{array}$ & $\begin{array}{l}62.5 \\
37.5 \\
\end{array}$ & $\begin{array}{l}13 \\
18 \\
\end{array}$ & $\begin{array}{l}41.9 \\
58.1 \\
\end{array}$ & $\begin{array}{l}24 \\
23 \\
\end{array}$ & $\begin{array}{l}51.1 \\
48.9 \\
\end{array}$ & $\begin{array}{l}2 \\
13 \\
\end{array}$ & $\begin{array}{l}13.3 \\
86.7 \\
\end{array}$ & 10.54 & 0.02 \\
\hline $\begin{array}{l}\text { Hb A1c } \\
<7 \% \\
\geq 7 \%\end{array}$ & $\begin{array}{l}18 \\
14\end{array}$ & $\begin{array}{l}56.3 \\
43.7 \\
\end{array}$ & $\begin{array}{l}18 \\
13\end{array}$ & $\begin{array}{l}58.1 \\
41.9\end{array}$ & $\begin{array}{l}12 \\
35\end{array}$ & $\begin{array}{l}25.5 \\
74.5\end{array}$ & $\begin{array}{l}0 \\
15\end{array}$ & $\begin{array}{l}0 \\
100\end{array}$ & 22.02 & 0.00 \\
\hline
\end{tabular}


(Table:3 ) Relationship of depression and diabetes self care, medication adherence and preventive care among the studied sample $(N=125)$

\begin{tabular}{|c|c|c|c|c|c|c|c|c|c|c|}
\hline Depression & Nor & mal & Mil & & Mo & erate & Sev & & Chi- & $\mathbf{P}$ \\
\hline Variable & No & $\%$ & No & $\%$ & No & $\%$ & No & $\%$ & & \\
\hline $\begin{array}{l}\text { Compliance to diet } \\
\text { restriction } \\
\text { Poor } \\
\text { Good }\end{array}$ & $\begin{array}{l}6 \\
26 \\
\end{array}$ & $\begin{array}{l}18.8 \\
81.2\end{array}$ & $\begin{array}{l}15 \\
16 \\
\end{array}$ & $\begin{array}{l}48.4 \\
51.6 \\
\end{array}$ & $\begin{array}{l}33 \\
14 \\
\end{array}$ & $\begin{array}{r}70.2 \\
29.8 \\
\end{array}$ & $\begin{array}{l}15 \\
0\end{array}$ & $\begin{array}{l}100 \\
0\end{array}$ & 34.23 & 0.00 \\
\hline $\begin{array}{l}\text { Compliance to } \\
\text { physical activity } \\
\text { Poor } \\
\text { Good }\end{array}$ & $\begin{array}{l}4 \\
28\end{array}$ & $\begin{array}{l}12.5 \\
87.5\end{array}$ & $\begin{array}{l}11 \\
20\end{array}$ & $\begin{array}{l}35.5 \\
64.5\end{array}$ & $\begin{array}{l}28 \\
19\end{array}$ & $\begin{array}{l}59.6 \\
40.4\end{array}$ & $\begin{array}{l}15 \\
0\end{array}$ & $\begin{array}{l}100 \\
0\end{array}$ & 36.88 & 0.00 \\
\hline $\begin{array}{l}\text { Compliance to } \\
\text { treatment on time } \\
\text { Poor } \\
\text { Good }\end{array}$ & $\begin{array}{l}1 \\
31\end{array}$ & $\begin{array}{l}3.1 \\
96.9\end{array}$ & $\begin{array}{l}5 \\
26\end{array}$ & $\begin{array}{l}16.1 \\
83.9\end{array}$ & $\begin{array}{l}26 \\
21\end{array}$ & $\begin{array}{l}55.3 \\
44.7\end{array}$ & $\begin{array}{l}12 \\
3\end{array}$ & $\begin{array}{l}80 \\
20\end{array}$ & 40.92 & 0.00 \\
\hline $\begin{array}{l}\text { Compliance to foot } \\
\text { check } \\
\text { Poor } \\
\text { Good }\end{array}$ & $\begin{array}{l}13 \\
19 \\
\end{array}$ & $\begin{array}{l}40.6 \\
59.4\end{array}$ & $\begin{array}{l}18 \\
13\end{array}$ & $\begin{array}{l}58.1 \\
41.9 \\
\end{array}$ & $\begin{array}{l}29 \\
18\end{array}$ & $\begin{array}{l}61.7 \\
38.3 \\
\end{array}$ & $\begin{array}{l}12 \\
3\end{array}$ & $\begin{array}{l}80 \\
20 \\
\end{array}$ & 7.18 & 0.07 \\
\hline $\begin{array}{l}\text { Compliance to check } \\
\text { inside shoes } \\
\text { Poor } \\
\text { Good }\end{array}$ & $\begin{array}{l}21 \\
11\end{array}$ & $\begin{array}{l}65.6 \\
34.4\end{array}$ & $\begin{array}{l}18 \\
13\end{array}$ & $\begin{array}{l}58.1 \\
41.9\end{array}$ & $\begin{array}{l}30 \\
17\end{array}$ & $\begin{array}{l}63.8 \\
36.2\end{array}$ & $\begin{array}{l}12 \\
3\end{array}$ & $\begin{array}{l}80 \\
20\end{array}$ & 2.17 & 0.54 \\
\hline $\begin{array}{l}\text { Compliance to test as } \\
\text { advised } \\
\text { Poor } \\
\text { Good }\end{array}$ & $\begin{array}{l}16 \\
16\end{array}$ & $\begin{array}{l}50 \\
50\end{array}$ & $\begin{array}{l}22 \\
9\end{array}$ & $\begin{array}{l}71 \\
29\end{array}$ & $\begin{array}{l}25 \\
22\end{array}$ & $\begin{array}{l}53.2 \\
46.8\end{array}$ & $\begin{array}{l}6 \\
9\end{array}$ & $\begin{array}{l}40 \\
60\end{array}$ & 4.95 & 0.18 \\
\hline $\begin{array}{l}\text { Compliance to bl. } \\
\text { glucose check } \\
\text { Poor } \\
\text { Good }\end{array}$ & $\begin{array}{l}24 \\
8 \\
\end{array}$ & $\begin{array}{l}75 \\
25 \\
\end{array}$ & $\begin{array}{l}28 \\
3 \\
\end{array}$ & $\begin{array}{l}90.3 \\
9.7 \\
\end{array}$ & $\begin{array}{l}37 \\
10 \\
\end{array}$ & $\begin{array}{l}78.7 \\
21.3 \\
\end{array}$ & $\begin{array}{l}12 \\
3 \\
\end{array}$ & $\begin{array}{l}80 \\
20 \\
\end{array}$ & 2.64 & 0.45 \\
\hline
\end{tabular}


(Table: 4) Relationship between diabetic complications and depression among the studied group $(\mathbf{N}=\mathbf{1 2 5})$

\begin{tabular}{|c|c|c|c|c|c|c|c|c|c|c|}
\hline Depression & \multicolumn{2}{|c|}{ Normal } & \multicolumn{2}{|c|}{ Mild } & \multicolumn{2}{|c|}{ Moderate } & \multicolumn{2}{|c|}{ Severe } & \multirow{2}{*}{$\begin{array}{c}\text { Chi- } \\
\text { Square }\end{array}$} & \multirow{2}{*}{$\begin{array}{c}\mathbf{P} \\
\text { value }\end{array}$} \\
\hline Variable & No & $\%$ & No & $\%$ & No & $\%$ & No & $\%$ & & \\
\hline $\begin{array}{l}\text { Complications } \\
\text { No } \\
\text { Yes }\end{array}$ & $\begin{array}{l}11 \\
21\end{array}$ & $\begin{array}{l}34.4 \\
65.6\end{array}$ & $\begin{array}{l}2 \\
29 \\
\end{array}$ & $\begin{array}{l}6.4 \\
93.6 \\
\end{array}$ & $\begin{array}{l}1 \\
46\end{array}$ & $\begin{array}{l}2.1 \\
97.9\end{array}$ & $\begin{array}{l}0 \\
15\end{array}$ & $\begin{array}{l}0 \\
100\end{array}$ & 97.43 & 0.00 \\
\hline $\begin{array}{l}\text { Neuropathy } \\
\text { No } \\
\text { Yes }\end{array}$ & $\begin{array}{l}17 \\
15\end{array}$ & $\begin{array}{l}53.1 \\
46.9\end{array}$ & $\begin{array}{l}6 \\
25\end{array}$ & $\begin{array}{l}19.4 \\
80.6\end{array}$ & $\begin{array}{l}4 \\
43\end{array}$ & $\begin{array}{l}8.5 \\
91.5\end{array}$ & $\begin{array}{l}0 \\
15\end{array}$ & $\begin{array}{l}0 \\
100\end{array}$ & 27.76 & 0.00 \\
\hline $\begin{array}{l}\text { Cardiovascular } \\
\text { No } \\
\text { Yes }\end{array}$ & $\begin{array}{l}27 \\
5\end{array}$ & $\begin{array}{l}84.4 \\
15.6\end{array}$ & $\begin{array}{l}27 \\
4\end{array}$ & $\begin{array}{l}87.1 \\
12.9\end{array}$ & $\begin{array}{l}35 \\
12\end{array}$ & $\begin{array}{l}74.5 \\
25.5\end{array}$ & $\begin{array}{l}6 \\
9\end{array}$ & $\begin{array}{l}40 \\
60\end{array}$ & 14.04 & 0.003 \\
\hline $\begin{array}{l}\text { Retinopathy } \\
\text { No } \\
\text { Yes }\end{array}$ & $\begin{array}{l}26 \\
6\end{array}$ & $\begin{array}{l}81.3 \\
18.7\end{array}$ & $\begin{array}{l}16 \\
15\end{array}$ & $\begin{array}{l}51.6 \\
48.4\end{array}$ & $\begin{array}{l}26 \\
21\end{array}$ & $\begin{array}{l}55.3 \\
44.7\end{array}$ & $\begin{array}{l}3 \\
12\end{array}$ & $\begin{array}{l}20 \\
80\end{array}$ & 16.46 & 0.001 \\
\hline $\begin{array}{l}\text { Nephropathy } \\
\text { No } \\
\text { Yes }\end{array}$ & $\begin{array}{l}26 \\
6\end{array}$ & $\begin{array}{l}81.3 \\
18.7\end{array}$ & $\begin{array}{l}23 \\
8\end{array}$ & $\begin{array}{l}74.2 \\
25.8\end{array}$ & $\begin{array}{l}22 \\
25\end{array}$ & $\begin{array}{l}46.8 \\
53.2\end{array}$ & $\begin{array}{l}1 \\
14\end{array}$ & $\begin{array}{l}6.7 \\
93.3\end{array}$ & 28.99 & 0.000 \\
\hline $\begin{array}{l}\text { Hypertension } \\
\text { No } \\
\text { Yes }\end{array}$ & $\begin{array}{l}21 \\
11\end{array}$ & $\begin{array}{l}65.6 \\
34.4\end{array}$ & $\begin{array}{l}19 \\
12 \\
\end{array}$ & $\begin{array}{l}61.3 \\
38.7 \\
\end{array}$ & $\begin{array}{l}23 \\
24\end{array}$ & $\begin{array}{l}48.9 \\
51.1\end{array}$ & $\begin{array}{l}3 \\
12 \\
\end{array}$ & $\begin{array}{l}20 \\
80\end{array}$ & 9.77 & 0.02 \\
\hline $\begin{array}{l}\text { Sexual } \\
\text { dysfunction } \\
\text { No } \\
\text { Yes }\end{array}$ & $\begin{array}{l}27 \\
5\end{array}$ & $\begin{array}{l}84.4 \\
15.6\end{array}$ & $\begin{array}{l}28 \\
3\end{array}$ & $\begin{array}{l}90.3 \\
9.7\end{array}$ & $\begin{array}{l}34 \\
13\end{array}$ & $\begin{array}{l}72.3 \\
27.7\end{array}$ & $\begin{array}{l}3 \\
12\end{array}$ & $\begin{array}{l}20 \\
80\end{array}$ & 28.59 & 0.00 \\
\hline
\end{tabular}

(Table:5) Binary logistic regression analysis of variable associated with depression among type 2 diabetic patients

\begin{tabular}{|l|c|c|}
\hline Variables & Beta Coefficients & Sig. \\
\hline Neuropathy & 4.413 & 0.000 \\
\hline Age & -0.304 & 0.000 \\
\hline Retinopathy & 2.941 & 0.003 \\
\hline Sex & 1.532 & 0.025 \\
\hline Cardiac complications & 1.938 & 0.046 \\
\hline Nephropathy & -1.318 & 0.159 \\
\hline Hypertension & 0.377 & 0.587 \\
\hline Sexual dysfunction & -0.174 & 0.840 \\
\hline
\end{tabular}

\section{Discussion}

The World Health Organization (WHO) emphasizes the significance of the relationship between mental and physical health (Moussavi et al., 2007). The prevalence of depression among type 2 diabetics included in this study $(\mathrm{N}=125)$ was $74.4 \% \quad(24.8 \%, 37.6 \%$, and $12 \%)$ represents; mild, moderate, and sever depression respectively. This high rate may be due to that, most of depressed patients had diabetic complications (68\%). The data regarding the prevalence of 
depression in T2DM in EGYPT are scarce. Also, studies from USA, and UK reported the prevalence of depression in T2DM varying from 30 to 83 per cent (Raval et al., 2010). The reasons for increased prevalence and of depression in diabetes are still poorly understood. The general view is that the burden of living with diabetes and its complications plays an important role in the etiology of depression in diabetes (Penninx et al., 2007).

In the current study, diabetic patients with depression $(100 \%, 91.5 \%$, and $67.7 \%$ with sever, moderate, and mild depression respectively), were younger than 50 years old, who represent an age-working diabetic patients with depression (Nasser et al., 2009), which is common in developing countries compared to older age groups in developed countries (IDF, 2009). This may lead to significant impact not only on diabetes care, but also on productivity. Studies have found that depressed people with diabetes can have significant work disability and increased absence of work, twice as common compared to normal population (Kivimaki et al., 2009). This is attributed to that the majority of the studied group $(\mathrm{N}=95)$ were younger than 50 years old, narrow age range (40-60 Ys), and to avoid the health derangement that associated with the elderly. It is well reported that older patients face many challenges including isolation, more diseases and disabilities; hence making them more prone to developing psychological conditions (Ganatra et al., 2008).

The present study reported that, the likelihood of depression was higher in females than males $(86.7 \%$ vs.13.3\% P 0.001) which are consistent with other studies (Nasser et al., 2009). This is may be due to that female constitute $58.4 \%$ of the studied group. Also, women is influenced by adverse experiences, sociocultural roles, psychological attributes, biological factors including hormones and poor social support (Raval et al., 2010). In addition, women in Arab/Muslim countries are at paramount risk from mental health problems (passivity, dependence and emotional expression), allows them to be more emotional and extroversive in nature, in comparison to men (Khuwaja and Kadir, 2010).

Duration of diabetes is also associated with development of depression in this study and has been reported by other researchers as well (Iype et al., 2009). The possible explanation is that, increased duration of diabetes is known to significantly increase the risk of developing complications and health care expenditures; as a result such patients are more prone to develop psychological illnesses (Khowaja et al., 2007).

Depressive symptoms were higher in those treated with insulin $(51.6 \%, 61.7 \%$, and $100 \%$ for mild, moderate and sever depression respectively), which is similar to a recent survey ( $\mathbf{L i}$ et al., 2008). As primary care patients treated with insulin reported higher diabetes-related emotional distress compared with oral- or diettreated patients. Also, greater distress was largely explained by greater disease severity and self-care burdens. In order to improve diabetes-specific quality of life, clinicians should address patients' sense of worry and guilt, uncertain acceptance of diabetes diagnosis, and unclear treatment goals (Delahanty et al., 2007). Also, this is may be due mis-concept about insulin which delivered by other patients or through the physician themselves who delay insulin to last stages and with complications of diabetes. Moreover, delayed initiation of insulin in type 2 is common among physicians due to failure to act when drug intensification is needed; this delays the opportunity for improving metabolic control and thereby makes a substantial number of patients vulnerable to diabetic complications and its adverse outcomes, including depression (Phillips et al., 2001). So, the physician are responsible not only to change the attitude of the patients toward insulin but also to be aware that insulin must be introduced in a timely fashion, that is, as soon as the oral anti-diabetic agents fail to maintain A1C $<7.0 \%$ (Chiasson, 2009).

Our study revealed that, severity of depression increase with the number of total pills that used for treatment of DM and its associated co-morbidities, which explain the higher likelihood of depressive 
symptoms (Raval et al., 2010), reported similar results.

People with depression tend to focus on illness episodes and medical symptoms, and selectively recall negative or unpleasant events. Accordingly, the current study showed that, diabetic patients with depression frequently complaining of three or more diabetic symptoms $(35.5 \%, 55.3 \%$, and $100 \%$ for those with mild, moderate, and sever depression respectively).There is also evidence that painful symptoms, and functional limitations, can induce psychological distress and depression. For many patients, depression is associated with increased symptom burden, functional disability and medical costs, related to a chronic medical condition such as diabetes (Simon, 1996). This is especially important given the high rates of depression among persons with diabetes. Also, the increase in somatic symptoms can be explained by the seriousness with which people in a given culture view psychological stress as compared to physical illness (Okasha et al., 2005).

So, treating clinicians may be puzzled by patients with co-morbid chronic medical conditions and depression who report higher levels of physical symptoms than other patients with comparable disease severity. High levels of symptoms that do not correlate with physical or laboratory assessments should prompt the clinician to assess for depression, so that inappropriate testing or treatment recommendations are avoided (Ludman et al., 2004).

The present study showed that, depressed patients were more sedentary, less adherent to diet restriction and smoking, this may explain the greater waist circumference and higher BMI (93.3\% of obese with type $2 \mathrm{DM}$ are more likely to have sever depression). Also, (Kuhwaja et al., 2010), found that obesity has been consistently identified as an independent factor associated with depression. In large community study, depression was found to be more common among diabetic women especially if they were overweight and the body weight in these women was a predictor of depression more than diabetes it self (Nichols and
Brown , 2003). This is attributed to that, altered body image associated with obesity and co-morbidities further perpetuate the depression (Raval et al., 2010).

No significant difference between depressed diabetics and non-depressed one, as regard compliance to foot check, check inside shoes, self blood glucose monitoring, do test as advised by their physicians (e.g, HbA1c, renal function, and lipid profile), so there is no difference between them in use of DM monitoring and preventive services; this may be due to lack of education that delivered to the patients. While non depressed diabetics were more compliant to administer treatment on time .So depression not influence use of physician initiated services (Lin et al., 2004). So it was not surprising that those with depression had higher levels of HbA1c than those without depression. As, depressive symptoms have a negative impact on important parameters of diabetes self-care over time. While the extent to which treating depression in diabetics would result in improved selfcare remains unclear, it is likely that symptoms of depression such as decreased energy and concentration, increased feelings of hopelessness and worthlessness, and loss of pleasure and interest would negatively impact on patients' ability to adhere to the difficult self-care routines required to successfully manage type 2 diabetes. It is important to consider that symptoms of depression that occur in the context of diabetes have important relationships with the experience of the illness.

It was revealed that, depressed patients had high levels of $\mathrm{Hb} \mathrm{A} 1 \mathrm{c}(41.9 \%, 74.5 \%$, and $100 \%$ of mild, moderate, and sever depression respectively), which is an indicator of uncontrolled DM. The higher likelihood of poor glycemic control in the depressed than the non depressed was consistent with findings from other international studies (Zuberi et al., 2011). Also, the relation of $\mathrm{HbAlc}$ to complications of DM has been proved. Hence long-term follow-up of the DCCT and UK Prospective Diabetes Study (UKPDS) cohorts suggests that treatment to A1C targets below or around $7 \%$ in the years soon after the diagnosis of diabetes 
is associated with long-term reduction in risk of macro-vascular disease. Subgroup analyses of clinical trials such as the DCCT and UKPDS and the microvascular evidence from the Action in Diabetes and Vascular Disease Controlled Evaluation (ADVANCE) trial suggests a small but incremental benefit in microvascular outcomes with A1C (ADA, 2009).

Moreover, our study indicated that depression was higher in patients with diabetes -related complications $(93.6 \%$, $77.9 \%$, and $100 \%$ (mild, moderate, and sever depression respectively) vs. $65.6 \%$ (non-depressed), such as neuropathy, retinopathy, cardiovascular, nephropathy, sexual dysfunction, and hypertension. Also, a meta-analysis of 27 studies found a significant association between depression and a wide variety of diabetes complications (Degroot et al., 2007).The current research extends these results to an Arab sample in EGYPT. The possible explanation for above findings might be that depression and chronic psychological stress is known to activate the hypothalamic-pitutary-adrenal axis, stimulate the sympathetic nervous system, increase inflammatory and platelet aggregation responses and decrease insulin sensitivity (Danese et al., 2009), thereby contributing to poor glycaemic control and increasing the risk of complications. Depressed and anxious individuals are also less likely to comply with diabetes self-care recommendations and more likely to follow sedentary lifestyles, remain physically inactive, indulge in smoking and high fat diet (Khuwaja et al., 2010). In diabetes, multiple studies have also documented that depression is associated with poor glycemic control and poor self-care behaviors (Egede and Ellis, 2008). It is important to keep in mind that the direction of the relationship may also be in the opposite direction, in that severe complications may increase the likelihood of depressive or anxious symptoms (Sulaiman et al., 2010). The outcome of this study highlights the importance of assessing and treating co-morbid mental health concerns as part of a comprehensive management plan for diabetes (Williams et al., 2006). Treating both co-morbid illnesses simultaneously enhances the likelihood of a successful outcome due to synergistic effects wherein the treatment of one condition affects the course of the other (Kinder et al., 2006).

By analyzing factors possibly affecting depression among type 2 diabetics, it was revealed that, being female, older in age and having neuropathy, retinopathy or cardiovascular complications, significantly and independently predicted depression.

The challenges in relation to screening, diagnoses and treatment of mental health problems in this, is that mental illness and the utilization of mental health services are highly stigmatized in the culture. Many also believe that a mental illness may be divine punishment as a result of disobedience or $\sin$, or due to weak faith (Suliaman et al., 2010).

\section{Conclusion and Recommendation}

The inclusion of established depression screening protocols in diabetes clinical management pathways would increase provider awareness, screening, and psychological referral. These steps could result in earlier detection and initiation of depression treatment. These steps can also facilitate open dialogue between patients and their providers to overcome the attitudinal and logistical barriers to depression treatment and encourage careful monitoring of patients beyond treatment to reduce the potential impact of depression relapse.

\section{References}

-ADA American Diabetes Association (2009) Standards of medical care in diabetes - 2009. Diab Care 32(Suppl 1):S13-S61.

-Chiasson JL (2009). Early Insulin Use in Type 2 Diabetes .what are the cons?_Diabetes Care November: vol. 32 no. suppl 2.

-Danese A, Moffitt TE, Harrington H, Milne BJ, Polanczyk G, Pariante CM, Poulton R, and Caspi A (2009). Adverse childhood experiences and adult risk factors for agerelated disease: depression, inflammation, and clustering of metabolic risk markers. Arch Pediatr Adolesc Med;163(12):1135-43.

-De groot, Hockman $\mathrm{E} M$ andDoyle T(2007). Depression among Type 2 Diabetes Rural Appalachian Clinic Attendees. Diabetes Care: 30: 1602-4. 
-Delahanty LM, Grant RW, Wittenberg E, Bosch JL, Wexler DJ, Cagliero E, and Meigs JB (2007). Association of diabetesrelated emotional distress with diabetes treatment in primary care patients with Type 2 diabetes. Diabetic Medicine: Volume 24, Issue 1, pages 48-54.

-Egede LE, and Ellis C (2008). The effects of depression on diabetes knowledge, diabetes self-management, and perceived control in indigent patients with type 2 diabetes. Diabetes Technol Ther;10 (3):213-219.

-Fisher L, Skaff MM, Mullan JT, Arean P, Glasgow R, and Masharani U (2008). A longitudinal study of affective and anxiety disorders, depressive affect and diabetes distress in adults with type 2 diabetes. Diabet Med; 25:1096-1101.

Ganatra HA, Zafar SN, Qidwai W, Rozi S (2008). Prevalence and predictors of depression among an elderly population of Pakistan. Aging Ment Health; 12:349-356.

-Geffken GR, Ward HE, Staab JP, Carmichael SL, and Evans DL (1998). Psychiatric morbidity in endocrine disorders. Psychiatr Clin North Am; 21:473-489.

-Gonzalez JS, Safren SA, Cagliero E, Wexler DJ, Delahanty L, Wittenberg E, Blais MA, Meigs JB, and Grant RW (2007). Depression, Self-Care, and Medication Adherence in Type 2 Diabetes: Relationships across the full range of symptom severity. Diabetes Care: 9:2222-7.

-Government of Egypt Ministry of Health Information Documentation Basic Health Data (1994). Cairo Government of Egypt.

-Herman WH, Ali MA, and Aubert RE (1995). Diabetes mellitus in Egypt: risk factors and prevalence. Diabet Med; 2(12):1126-31.

-Herman WH,Aubert RE,Ali MA ,Sous ES, and Badran A(1997). Diabetes mellitus in Egypt. Risk factors prevalence and future burden. Eastren Mediterranean health Journal: vol 3, issue1, p 144-148.

-International Diabetes Federation (2009). www.eatlas.idf.org. Accssed on 3.7 .

-Iype T, Shaji SK, Balakrishnan A, Charles D, Varghese A, and Antony TP (2009). Cognition in type 2 diabetes: association with vascular risk factors, complications of diabetes and depression. Ann Indian Acad Neurol; 12:25-27.

-Katon W (2010). Depression and Diabetes. Unhealthy bedfellows. Depression And Anxiety 27:323-326.

-Khowaja LA, Khuwaja AK, and Cosgrove P (2007). Cost of diabetes care in out-patient clinics of Karachi, Pakistan. BMC Health Serv Res; 21:189.
-Khuwaja AK, and Kadir MM (2010). Gender differences and clustering patterns of behavioral risk factors for chronic noncommunicable diseases: community-based study from a developing country. Chronic Illn; 6(3):163-170.

-Khuwaja AK, Lalani S, Dhanani R, Azam IS, Rafique G, and White F(2010). Anxiety and depression among outpatients with type 2 diabetes: A multi-centre study of prevalence and associated factors. Diabetol Metab Syndr.,2:72.

-Kinder L, Katon W, Ludman E, Russo J, Simon G, Lin , Ciechanowski P, Von Korff M, and Young B (2006). Improving depression care in patients with diabetes and multiple complications. J Gen Intern Med; 21(10):1036-1041.

-King H, Aubert RE, and Herman WH (1998). Global burden of diabetes, 1995-2025: prevalence, numerical estimates, and projections. Diabetes Care; 21(9):1414-31.

-Kivimaki M, Vahtera J, and Pentti J (2009). Increased Sickness Absence in Diabetic Employees: What Is the Role of CoMorbid Conditions? Diabetic Medicine: 24(9): 1043-48.

-Lester H, and Howe A (2008). Primary care research ,depression in primary care: the key challenges .Post grade Med J; 84:545-548.

-Li C, Ford ES, and Strine TW (2008). Prevalence of Depression among U.S Adults with Diabetes. Findings from the 2006 Behavioral Risk Factor Surveillance System. Diabetes Care; 31: 105-7.

-Lin E, Heckbert S, Rutter C, Katon W, Ciechanowski P, Ludman E, Oliver M, Young B, McCulloch DK, and Von Korff $M$ (2009). Depression and increased mortality in diabetes: unexpected causes of death. Ann Fam Med; 7: 414- 42.

-Lin EH, Katon W, Von Korff M, Rutter C, Simon GE, Oliver M, Ciechanowski P, Ludman EJ, Bush T, and Young B (2004). Relationship of depression and diabetes selfcare, medication adherence, and preventative care. Diabetes Care; 27:2154-2160.

-Ludman EJ, Katon W, Russo J, Von Korff M, Simon G, Ciechanowski P, Lin E, Bush T, Walker E, and Young B (2004). Depression and diabetes symptom burden. Gen Hosp Psychiatry; 26: 430- 436.

-Markowitz S, Gonzalez JS, Wilkinson JL, and Safren SA (2011). Treating Depression in Diabetes: Emerging findings. Psychosomatics; Jan-Feb; 52(1): 1-18.

-Montgomery SA, and Asberg M (1979). A new depression scale designed to be sensitive to change. British Journal of Psychiatry 134 (4): $382-89$. 
-Moussavi S, Somnath C, Verdes E, Tandon A, Patel V, and Ustun B (2007). Depression, chronic diseases, and decrements in health: results from the World Health Surveys. Lancet; 370(9590):851-858.

-Nasser J, Habib F, Hasan M, and Khalil N (2009). Prevalence of depression among people with diabetes attending diabetes clinic at primary health settings. Bahrain Medical Bulletin; 1.31(3):212-16.

-Nichols GA, and Brown JB (2003). Unadjusted and Adjusted Prevalence of Diagnosed Depression in Type 2 Diabetes. Diabetes Care; 26: 744-49.

-Okasha A (2005). Mental Health in Egypt. WHO Collaborating Center for Training and Research, Institute of Psychiatry, Ain Shams University, Kasr El-Nile, Cairo, Egypt. Isr J Psychiatry Relat Sci Vol 42 No. 2 116-125.

-Penninx BW, Beekman AT, and Bandinelli S (2007). Late-life depressive symptoms are associated with both hyperactivity and hypoactivity of the hypothalamo-pituitary-adrenal axis. Am J Geriatr Psychiatry 15:522-529.

-Phillips LS, Branch W, and Cook CB (2001). Clinical Inertia. Ann Intern Med; 135: 825-34.

-Pouwer F, Geelhoed-Duijvestijn PHLM, Tack Cj, Bazelmans E, Beekman AJ, Heine RJ, and Snoek FJ (2010). Prevalence of comorbid depression is high in out - patients with type 1 or type 2 diabetes mellitus. Results from three out -patient clinics in the
Netherlands. Diabetic Medicine vol 27,issue 2,pages217-224.

-Raval A, Dhanaraj E, Bhansali A, Grover S, and Tiwari P (2010). Prevalence \& determinants of depression in type 2 diabetes patients in a tertiary care centre. Indian $\mathrm{J}$ Med Res 132, pp 195-200.

-Saman IZ, Ehsan US, and Junaid AB (2011). Association of depression with treatment outcomes in type 2 DM: A cross sectional study from Karachi, Pakistan. BMC Psychiatry; 11-27.

-Simon GE (1996). Psychiatric disorders and functional somatic symptoms as predictors of health care use. Psychiatr. Med;10:49-59.

-Sulaiman N , Hamdan A, Tamim H, Mahmood DA, and Young D (2010). The prevalence and correlates of depression and anxiety in a sample of diabetic patients in Sharjah, United Arab Emirates. BMC Family Practice; 11:80.

-Timonen M, and LiukkonenT (2008). Management of depression in adults BMJ vol 336, NO, 7641; 336:435.

-Whitty P, Steen N, Eccles M (1997). A new completion outcome measure for diabetes: is it responsive to change?. Qual. Life Res; 6:40741.

-Williams M, Clouse R, and Lustman $\mathbf{P}$ (2006). Treating depression to prevent diabetes and its complications: understanding depression as a medical risk factor. Clin Diabetes; 24(2):79-86. 


\title{
الاكتئاب بين المرضى المصابين بمرض السكرى النوع الثانى
}

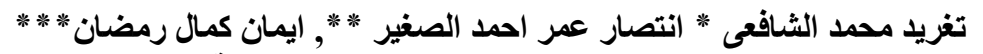

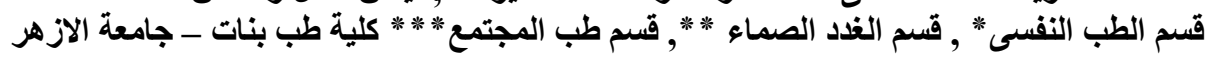

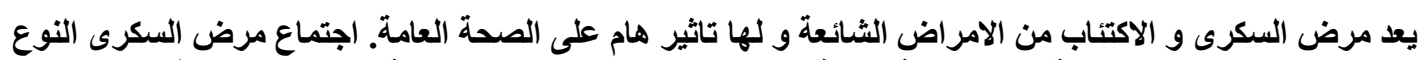

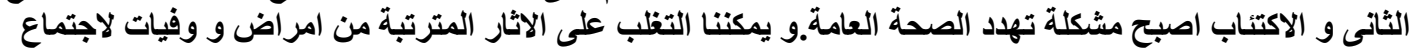

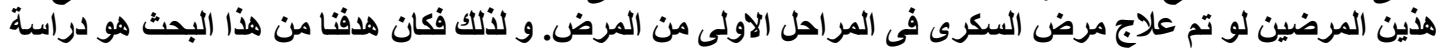

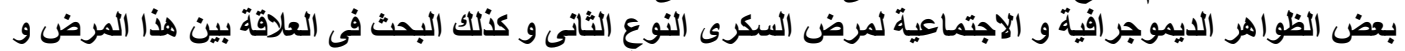

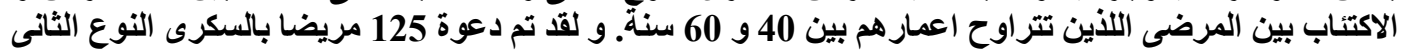

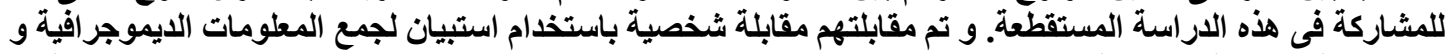

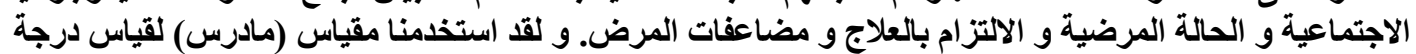

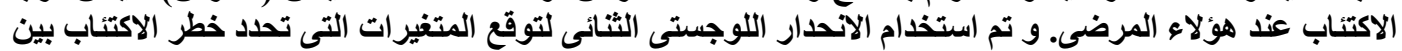
المرضى المصابين بمرض السكرى النوع الثانى و ذلك عند فاصل الثقة 95\% و و الهمية احصائية اقل من او يساوى .05.

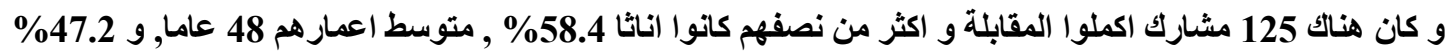

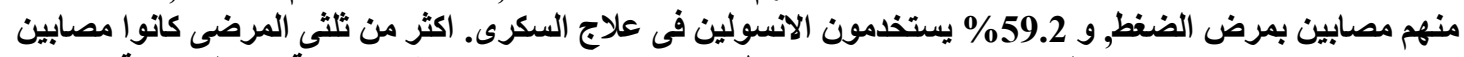

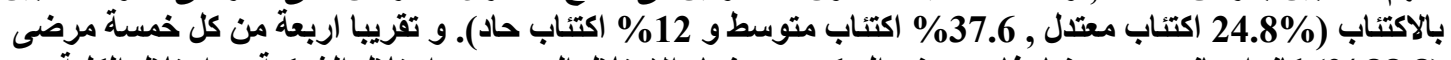

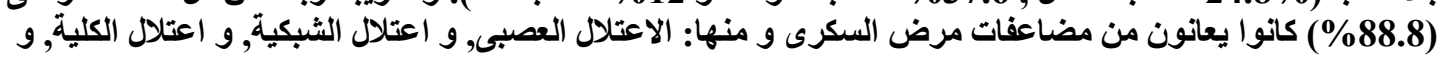

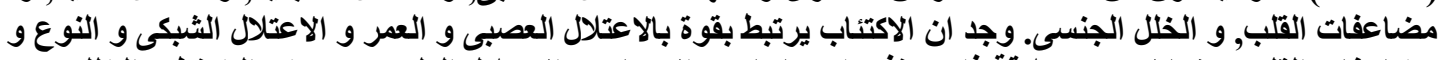

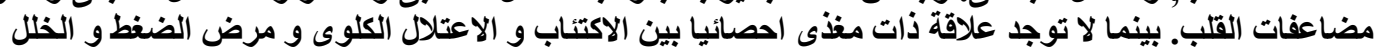
الجنسى.

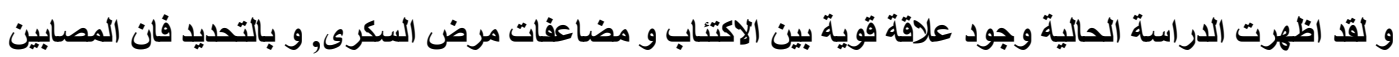

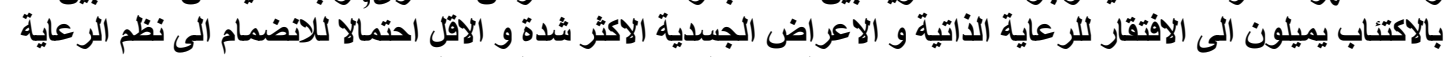

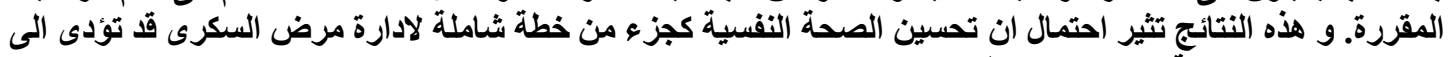

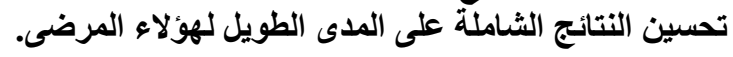

